\title{
The effect of orlistat versus metformin on body composition and insulin resistance in obese premenopausal women: 3-month randomized prospective open-label study
}

Magdalena Kujawska-Łuczak ${ }^{1}$, Katarzyna Musialik², Monika Szulińska², Ewelina Swora-Cwynar ${ }^{3}$, Angelina Kargulewicz ${ }^{3}$, Małgorzata Grzymisławska ${ }^{4}$, Danuta Pupek-Musialik ${ }^{1}$, Paweł Bogdański²

\author{
${ }^{1}$ Department of Internal Medicine, Metabolic Disorders, and Hypertension, \\ Poznan University of Medical Sciences, Poznan, Poland \\ 2Department of Education and Obesity Treatment and Metabolic Disorders, \\ Poznan University of Medical Sciences, Poznan, Poland \\ ${ }^{3}$ Department of Internal Medicine, Metabolic Disorders and Dietetics, \\ Poznan University of Medical Sciences, Poznan, Poland \\ ${ }^{4}$ Department of Anatomy, Poznan University of Medical Sciences, Poznan, Poland
}

Submitted: 21 February 2016

Accepted: 19 June 2016

Arch Med Sci 2017; 13, 4: 725-731

DOI: https://doi.org/10.5114/aoms.2016.62014

Copyright (c) 2016 Termedia \& Banach

\section{Abstract}

Introduction: Our aim was to evaluate the effects of metformin and orlistat on body composition and glucose-insulin homeostasis in obese premenopausal women.

Material and methods: Seventy-three obese premenopausal Caucasian women aged $32.4 \pm 8.3$ years were treated with either metformin $(1000 \mathrm{mg} /$ day; $n=37$ ) or orlistat (360 mg/day; $n=36$ ). Anthropometric parameters were measured using dual-energy X-ray absorptiometry. Glucose tolerance, using the oral glucose tolerance test; insulin resistance, using the homeostasis model assessment (HOMA-IR); and insulin sensitivity, using the Matsuda insulin sensitivity index (ISI Matsuda), were assessed at the commencement of the study and after 3 months.

Results: Those treated with orlistat showed greater weight loss $(-9.4 \pm 2.3 \mathrm{vs}$. $-4.9 \pm 1.3 \mathrm{~kg}, p<0.05)$ and decrease of fat mass $(-5.4 \pm 3.0 \mathrm{vs} .-3.5 \pm 0.7 \mathrm{~kg}$, $p<0.05)$ than those treated with metformin. The percentage of android and gynoid fat deposits was reduced in both groups; however, a greater decrease in android fat was observed in those treated with metformin. Improvement in ISI Matsuda and post-load insulin were similar in both groups. High initial post-load insulin and low ISI Matsuda corresponded with reductions in total fat, trunk fat, and waist circumference in both groups, and a decrease in android fat in those treated with metformin.

Conclusions: Orlistat treatment resulted in greater weight loss and improvement in body composition; metformin treatment resulted in a reduction of android fat. Both drugs produced a comparable improvement in insulin/glucose homeostasis. Overall, insulin-resistant women showed improvement with treatment, irrespective of which drug was used.

Key words: orlistat, metformin, obesity, dual-energy densitometry, insulin resistance.
Corresponding author: Magdalena Kujawska-Łuczak MD

Department of Internal Medicine, Metabolic Disorders and Hypertension Poznan University of Medical Sciences 84 Szamarzewskiego St 60-569 Poznan, Poland Phone: +48600243068 E-mail: magaluczak@wp.pl 


\section{Introduction}

Obesity is becoming an increasingly serious problem in Poland, especially among women [1]. Abdominal obesity is associated with numerous health complications, including insulin resistance (IR), type 2 diabetes, cardiovascular disease, and increased mortality [2, 3]. It is well known that obese premenopausal women receive significant health benefits as a result of weight reduction, as well as from a reduction of abdominal obesity with concomitant improvement of IR.

Dual-energy densitometry (DXA) is one of the recommended methods of assessment of body fat percentage, as well as android and gynoid fat and the ratio between the two $(A: G)[4,5]$. It has also been used to assess the effects of weight reduction [6]. Android fat and A : G ratio have each been shown to correlate with IR, risk of myocardial infarction and mortality [7-9].

Metformin is an insulin-sensitizer, recommended for use by pre-diabetic and type 2 diabetic patients. Recently, metformin was demonstrated to aid in weight reduction in insulin-sensitive, as well as insulin-resistant, overweight and obese patients $[10,11]$. Treatment with metformin has been associated with a significant reduction in visceral (VAT) and subcutaneous adipose tissue (SAT) in obese women, and an increase in insulin sensitivity $[12,13]$. Orlistat decreases the absorption of ingested lipids by inhibiting gastric and pancreatic lipase. Previous studies have shown that orlistat treatment results in significant weight loss and a decrease in waist circumference $[14,15]$. Treatment with orlistat has also been shown to improve insulin sensitivity and the ratio between intra-abdominal and subcutaneous fat $[16,17]$.

To our best knowledge, to date no studies have assessed the individual effects of treatment with metformin and orlistat on glucose-insulin homeo-

Table I. Dietary composition during the study

\begin{tabular}{|lc|}
\hline Dietary component & $\begin{array}{c}\text { Percent of total } \\
\text { energy intake }\end{array}$ \\
\hline \begin{tabular}{lc} 
Carbohydrate intake, including: \\
\hline Complex carbohydrates
\end{tabular} & $50-55$ \\
\hline Saccharose & $45-50$ \\
\hline Protein intake & $20-25$ \\
\hline Fat intake, including: & 7 \\
\hline $\begin{array}{l}\text { Saturated fatty acids } \\
\text { Monounsaturated fatty acids }\end{array}$ & 10 \\
\hline $\begin{array}{l}\text { Polyunsaturated fatty acids } \\
\text { Cholesterol intake [mg/day] }\end{array}$ & 8300 \\
\hline
\end{tabular}
obese non-diabetic premenopausal women. It remains to be investigated which of these two treatments is superior in terms of DXA measurements.

This study was designed to evaluate the individual effects of treatment with metformin and orlistat on body composition and insulin resistance in obese non-diabetic premenopausal women.

\section{Material and methods}

The study was approved by the Ethics Committee of Poznan University of Medical Sciences. The trial protocol met the requirements of the Declaration of Helsinki.

We conducted a prospective randomized openlabel study assessing the effects of treatment with metformin (M) and orlistat (O) on obesity. Seventy-three women were enrolled; they were recruited from 2010 to 2013 from referrals to the Department of Internal Medicine, Metabolism, and Dietetics and the Department of Internal Medicine, Metabolic Disorders, and Hypertension of Poznan University of Medical Sciences. The inclusion criteria were: age 18 to 40 years, body mass index (BMI) $\geq 30 \mathrm{~kg} / \mathrm{m}^{2}$ (obese), stable body weight for 1 month prior to the trial $( \pm 1 \mathrm{~kg})$, and Caucasian. We excluded patients who: were diagnosed with acute or chronic disease (including diabetes), smoked tobacco, drank more than two alcoholic drinks per week, were addicted to drugs, pregnant or gave birth in the 3 months prior to enrolment, currently lactating or were lactating in the 3 months prior to enrolment, and those who had entered menopause. All included patients underwent a 12-week run-in period, which started with the acquisition of dietary advice, including a structured weight-maintenance diet, from a qualified dietician (Table I). A qualified dietician monitored the patients' dietary intake on the basis of interviews and food diaries, every 14 days until the end of the trial. The intake of nutrients, total calories, and caffeine was maintained at a constant level during the study. Each patient received a personalized food plan from her dietician. During the trial, the consumption of dietary supplements was not recommended.

After a 12-week run-in period, no significant changes in weight in either group M (109.3 $\pm 23.8 \mathrm{~kg}$ vs. $108.9 \pm 24.1 \mathrm{~kg})$ or group O (103.2 $\pm 23.7 \mathrm{~kg}$ vs. $103.0 \pm 24.1 \mathrm{~kg}$ ) were observed. After the run-in period, all women were subjected to a 3-month drug intervention aimed at reduction of body weight. Women aged $31.4 \pm 8.2$ years were randomized to one of two study groups: metformin-treated (group $M$ ) or orlistat-treated (group 0$)$. In group $M(n=37)$, individual isocaloric diets were combined with the twice-daily administration of $500 \mathrm{mg}$ of M. In group O $(n=36)$, individual isocaloric diets were combined with the 
administration of $120 \mathrm{mg}$ of $\mathrm{O}$, three times a day. Compliance was monitored based on counting returned medication (number of pills). All subjects completed the 3-month study. Two patients in group $M$ showed symptoms of nausea and mild abdominal pain, and three patients in group $\mathrm{O}$ showed oily stools and cramping. As these symptoms were not classified as severe, there was no need to reduce the doses of drugs or stop the treatment.

All subjects were instructed to maintain their usual physical activity regimen throughout the 3-month study. Under basal conditions and 3 months after the beginning of the trial, a number of parameters were assessed, namely, anthropometric measurements, fat tissue content, body composition, and serum concentrations of glucose and insulin (fasting and 120 min oral glucose tolerance test - OGTT).

\section{Anthropometric parameters}

Body mass and height were measured the morning after $14 \mathrm{~h}$ of overnight fasting, with an accuracy of $0.1 \mathrm{~kg}$ and $0.5 \mathrm{~cm}$, respectively. Body mass index was calculated using the standard formula (weight $[\mathrm{kg}] /$ height $^{2}\left[\mathrm{~m}^{2}\right]$ ). Obesity was diagnosed according to the criteria set by the World Health Organization. Waist circumference was measured to the nearest $0.5 \mathrm{~cm}$ at the point midway between the uppermost border of the iliac crest and the lower border of the costal margin (the end of normal expiration).

\section{Body composition using dual-energy X-ray absorptiometry}

Fat tissue content was assessed using dual-energy X-ray absorptiometry (GE Healthcare Lunar Prodigy Advance; GE Medical Systems; Italy). The DXA machine was reset each day according to the standard procedure, and spine and anthropomorphic phantoms were scanned daily. The same laboratory technician performed all scans and analysis, according to the operator's manual. The intrasubject and intersubject coefficients of variation $(\mathrm{CV} \%=100 \times \mathrm{SD} /$ mean $)$ ranged from $1 \%$ to $5 \%$. Total and regional scans were taken. Total and trunk fat were measured, as were android and gynoid fat distribution using specific anatomical landmarks. A:G and trunk to total fat ratios were calculated as measures of abdominal obesity.

\section{Oral glucose tolerance test}

Glucose concentrations were measured using blood samples drawn after a 14-h overnight fast and $2 \mathrm{~h}$ after oral administration of $75 \mathrm{~g}$ of anhydrous glucose dissolved in $200 \mathrm{ml}$ of water. Samples of venous blood were drawn from a forearm vein. The measurement of plasma glu- cose concentration was performed using the Glucose HK Gen.3 enzymatic assay with hexokinase (Roche Diagnostics, Mannheim, Germany) and a Cobas Integra analyzer (Roche, Basel, Switzerland). The reference range of the assay was 0.12$40.0 \mathrm{mmol} / \mathrm{l}$. The plasma insulin concentrations were measured using an electrochemiluminescent immunoassay (ECLIA, Roche Diagnostics, Mannheim, Germany) with a lower limit of sensitivity of $0.20 \mu \mathrm{U} / \mathrm{ml}$ and intra- and interassay coefficients of variations $2.1-2.8 \%$ and $1.4-2.8 \%$, respectively.

Insulin resistance (IR) was calculated using the homeostatic model for assessment of insulin resistance $(\mathrm{HOMA})$ method: $\mathrm{HOMA}-\mathrm{IR}=$ fasting glucose concentration $[\mathrm{mmol} / \mathrm{l}] \times$ fasting insulin concentration $[\mathrm{mU} / \mathrm{l}] / 22.5$ [18].

The insulin sensitivity index of Matsuda (ISI Matsuda) was calculated as 10,000 divided by the square root of ((fasting glucose $\times$ fasting insulin $) \times(120$ glucose $\times 120$ insulin during OGTT $))$ $(10,000 /(\mathrm{mmol} / \mathrm{l} \times \mathrm{mU} / \mathrm{l}))$. This was highly correlated $(r=0.651, p<0.0001)$ with the rate of whole-body glucose disposal during the euglycemic insulin clamp [19]. ISI Matsuda, measured using both fasting and 120-min glucose and insulin concentrations during the OGTT, is considered to reflect both liver and muscle sensitivity and correspond better with metabolic disturbances [20]. To identify women with IR we used the cut-off proposed by Radikova et al. [21]. In most papers ISI Matsuda was calculated using glucose in $\mathrm{mg} / \mathrm{dl}$. As we used SI units for glucose [mmol/l], to obtain comparable results of ISI Matsuda we divided the results obtained from the formula by 18 . The threshold proposed by Radikova (5.0) allowed us to identify women with IR.

\section{Statistical analysis}

The data are presented as mean \pm SD. All calculations and demographics were performed using the Statistica 10 software (StatSoft, Inc., 2011). All changes before and after the intervention were calculated as differences between the final and initial values (with negative values indicating a decrease). The Shapiro-Wilk test was used to check the normal distribution. Comparisons between groups were assessed using the Mann-Whitney $U$ test, or the unpaired $t$-test if the data were normally distributed. The Wilcoxon rank-sum test or the paired $t$-test (for data with normal distribution) were used to analyze the statistical difference between variables before and after the intervention. Insulin, HOMA-IR, and ISI Matsuda were logarithmically transformed to be normally distributed, then Pearson's correlation test was used to calculate the correlations between analyzed parameters. The $\chi^{2}$ test was used to compare the IR rate in both groups. A $p$-value of 
less than 0.05 was regarded as significant. It was determined that the sample size of a minimum of 30 subjects in each group would yield at least an $80 \%$ power of detecting an effect as statistically significant at the $0.05 \alpha$ level.

\section{Results}

\section{Body composition measurements}

Changes in body composition measurements are summarized in Table II. The reduction of body mass was significant in both groups after 3 months of pharmacological treatment but was significantly greater in group $\mathrm{O}$ than in group $\mathrm{M}$. Our results also showed significant and comparable decreases in waist circumference, as well as a reduction in total body fat. When the two groups were directly compared, the decreases in body fat (\% and $\mathrm{kg}$ ) were all markedly greater in group 0 . DXA measurements revealed comparable reductions in android and gynoid fat in both groups; however, greater decreases in android and trunk fat, measured in $\mathrm{kg}$, were seen in group $M$.

\section{Measures of insulin resistance and sensitivity}

Table III shows a summary of values of insulin-derived parameters in both examined groups. On the basis of ISI Matsuda we identified women with IR at the start of the intervention. There were 6 in group $M$ and 9 in group $O$. These numbers were comparable. Neither treatment was observed to affect fasting insulin concentrations. We observed significant decreases in post-challenge 120-min insulin levels in both groups $M$ and $\mathrm{O}$. We also found a slight, though not significant, improvement in HOMA-IR in both groups. We observed improvement in ISI Matsuda in both groups. Additionally, both groups were observed to have high initial post-load insulin and low ISI Matsuda concomitantly with large reductions in waist circumference after treatment. Both parameters also strongly correlated with decreases in total and trunk fat in both groups, and android fat in group O (Table IV). Stronger correlations between initial insulin resistance status and changes in abdominal adiposity were found in group $M$.

\section{Discussion}

After 3 months of treatment, we observed significant decreases in body weight in both groups, but the decrease observed in the orlistat group was twice as large. Reductions in waist circumference, body fat, and percentage of android and gynoid fat were similar in both groups. When the two groups were compared, significantly greater decreases in android and trunk fat (measured in kg) were seen in the metformin group. In previous studies of obese women, metformin treatment significantly decreased the amount of visceral and subcutaneous fat $[12,13]$. Orlistat treatment also resulted in significant improvements in body composition and had a positive effect on the intra-ab-

Table II. Anthropometric measurements and body composition: patient characteristics at baseline and after 3 months of treatment

\begin{tabular}{|c|c|c|c|c|c|c|c|}
\hline \multirow[t]{2}{*}{ Parameter } & \multicolumn{3}{|c|}{ Group M } & \multicolumn{3}{|c|}{ Group 0} & \multirow{2}{*}{$\begin{array}{c}\text { Treatment } \\
\text { difference } \\
p<0.05\end{array}$} \\
\hline & Baseline & $\begin{array}{c}\text { After } \\
\text { treatment }\end{array}$ & Change & Baseline & $\begin{array}{c}\text { After } \\
\text { treatment }\end{array}$ & Change & \\
\hline \multicolumn{8}{|l|}{ Anthropometry: } \\
\hline Weight [kg] & $108.9 \pm 24.1$ & $104.0 \pm 23.7$ & $-4.9 \pm 1.3^{*}$ & $103.3 \pm 24.1$ & $93.7 \pm 20.1$ & $-9.4 \pm 2.3^{*}$ & M vs. O \\
\hline $\mathrm{BMI}\left[\mathrm{kg} / \mathrm{m}^{2}\right]$ & $40.1 \pm 8.1$ & $38.2 \pm 8.1$ & $-1.7 \pm 0.4^{*}$ & $37.0 \pm 4.6$ & $33.4 \pm 6.0$ & $-3.2 \pm 0.8^{\star}$ & M vs. O \\
\hline Waist $[\mathrm{cm}]$ & $110.2 \pm 5.6$ & $105.1 \pm 16.6$ & $-5.8 \pm 1.5^{\star}$ & $110.3 \pm 12.4$ & $99.4 \pm 12.8$ & $-10.5 \pm 2.5^{\star}$ & NS \\
\hline \multicolumn{8}{|l|}{ DXA measurements: } \\
\hline Android fat (\%) & $55.8 \pm 4.8$ & $51.9 \pm 4.6$ & $-3.9 \pm 0.9^{*}$ & $54.0 \pm 4.1$ & $47.7 \pm 4.0$ & $-6.2 \pm 4.2^{\star}$ & NS \\
\hline Android fat [kg] & $4.6 \pm 1.3$ & $3.8 \pm 1.2$ & $-0.7 \pm 0.2^{\star}$ & $4.7 \pm 0.8$ & $4.6 \pm 0.7$ & $-0.1 \pm 0.1$ & M vs. $O$ \\
\hline Gynoid fat (\%) & $54.5 \pm 3.6$ & $51.9 \pm 2.6$ & $-2.5 \pm 1.2$ & $52.5 \pm 3.6$ & $48.2 \pm 2.8$ & $-4.3 \pm 2.0^{\star}$ & NS \\
\hline Gynoid fat [kg] & $8.3 \pm 1.2$ & $7.6 \pm 1.2$ & $-0.6 \pm 0.1^{\star}$ & $8.4 \pm 1.9$ & $7.7 \pm 0.9$ & $-0.6 \pm 0.0^{\star}$ & NS \\
\hline Android/gynoid fat & $1.02 \pm 0.08$ & $1.00 \pm 0.06$ & $-0.02 \pm 0.02$ & $1.00 \pm 0.10$ & $0.90 \pm 0.09$ & $-0.12 \pm 0.03$ & NS \\
\hline Trunk fat $[\mathrm{kg}]$ & $26.4 \pm 6.4$ & $22.5 \pm 6.1$ & $-3.8 \pm 0.7^{\star}$ & $26.3 \pm 4.5$ & $25.7 \pm 4.0$ & $-0.72 \pm 0.68$ & M vs. $\mathrm{O}$ \\
\hline Trunk/total fat (\%) & $0.55 \pm 0.06$ & $0.50 \pm 0.06$ & $-0.04 \pm 0.04$ & $0.54 \pm 0.05$ & $0.53 \pm 0.04$ & $-0.01 \pm 0.03$ & NS \\
\hline Fat (\%) & $50.4 \pm 3.6$ & $48.1 \pm 2.8$ & $-2.2 \pm 0.9^{\star}$ & $48.9 \pm 3.3$ & $42.7 \pm 2.6$ & $-4.6 \pm 3.1^{\star}$ & M vs. O \\
\hline Fat $[\mathrm{kg}]$ & $47.6 \pm 8.0$ & $43.9 \pm 7.8$ & $-3.5 \pm 0.7^{\star}$ & $47.6 \pm 10.9$ & $40.1 \pm 8.5$ & $-5.4 \pm 3.0^{\star}$ & M vs. O \\
\hline
\end{tabular}


Table III. Glucose-insulin homeostasis: patient characteristics at baseline and after 3 months of treatment

\begin{tabular}{|c|c|c|c|c|c|c|c|}
\hline \multirow[t]{2}{*}{ Parameter } & \multicolumn{3}{|c|}{ Group M } & \multicolumn{3}{|c|}{ Group 0} & \multirow{2}{*}{$\begin{array}{c}\text { Treatment } \\
\text { difference } \\
p<0.05\end{array}$} \\
\hline & Baseline & $\begin{array}{c}\text { After } \\
\text { treatment }\end{array}$ & Change & Baseline & $\begin{array}{c}\text { After } \\
\text { treatment }\end{array}$ & Change & \\
\hline $\begin{array}{l}\text { Glucose } 0 \\
{[\mathrm{mmol} / \mathrm{l}]}\end{array}$ & $5.56 \pm 0.78$ & $5.65 \pm 0.72$ & $0.09 \pm 0.69$ & $5.23 \pm 0.72$ & $5.54 \pm 0.82$ & $0.31 \pm 1.06$ & NS \\
\hline $\begin{array}{l}\text { Glucose } 120 \\
{[\mathrm{mmol} / \mathrm{l}]}\end{array}$ & $7.60 \pm 1.61$ & $7.46 \pm 1.21$ & $-0.14 \pm 1.76$ & $7.16 \pm 2.35$ & $6.96 \pm 1.22$ & $-0.20 \pm 2.24$ & NS \\
\hline $\begin{array}{l}\text { Insulin } 0 \\
{[\mathrm{mU} / \mathrm{l}]}\end{array}$ & $19.9 \pm 19.1$ & $17.3 \pm 15.7$ & $-2.5 \pm 13.8$ & $11.9 \pm 5.7$ & $9.5 \pm 5.5$ & $-2.4 \pm 3.6$ & NS \\
\hline $\begin{array}{l}\text { Insulin } 120 \\
{[\mathrm{mU} / \mathrm{l}]}\end{array}$ & $101.5 \pm 65.6$ & $83.9 \pm 56.1$ & $-18.8 \pm 40.2^{*}$ & $78.5 \pm 62.1$ & $60.0 \pm 54.8$ & $-19.0 \pm 50.5^{\star}$ & NS \\
\hline HOMA-IR & $3.31 \pm 1.98$ & $2.96 \pm 1.90$ & $-0.38 \pm 2.56$ & $2.81 \pm 1.39$ & $2.23 \pm 1.28$ & $-0.58 \pm 0.96$ & NS \\
\hline ISI Matsuda & $53.8 \pm 10.8$ & $38.5 \pm 12.5$ & $17.5 \pm 47.4^{\star}$ & $99.3 \pm 10.5$ & $80.5 \pm 10.1$ & $18.6 \pm 10.3^{*}$ & NS \\
\hline
\end{tabular}

Data are presented as mean $\pm S D$. *Statistically significant differences $(p<0.05)$ between the initial and final values. $M-m e t f o r m i n ~ g r o u p$, $O$ - orlistat group, HOMA-IR - homeostatic model assessment-insulin resistance, ISI - insulin sensitivity index.

Table IV. Correlations between initial insulin resistance/sensitivity indices and 3-month changes in obesity parameters

\begin{tabular}{|c|c|c|c|c|c|c|}
\hline \multirow[t]{2}{*}{ Parameter } & \multicolumn{3}{|c|}{ Group M } & \multicolumn{3}{|c|}{ Group 0} \\
\hline & $\begin{array}{c}\text { Log } \\
\text { 120-insulin* }\end{array}$ & Log ISI & $\begin{array}{c}\log \\
\text { HOMA-IR }\end{array}$ & $\begin{array}{c}\text { Log } \\
\text { 120-insulin }\end{array}$ & $\begin{array}{l}\text { Log } \\
\text { ISI }\end{array}$ & $\begin{array}{l}\log \\
\text { HOMA-IR }\end{array}$ \\
\hline \multicolumn{7}{|l|}{ Anthropometry: } \\
\hline BMI $\left[\mathrm{kg} / \mathrm{m}^{2}\right]$ & NS & NS & NS & NS & NS & NS \\
\hline Waist [cm] & -0.40 & 0.38 & -0.30 & -0.51 & 0.40 & NS \\
\hline \multicolumn{7}{|l|}{ DXA measurements: } \\
\hline Fat (\%) & -0.37 & 0.56 & NS & -0.33 & 0.40 & NS \\
\hline Fat $[\mathrm{kg}]$ & -0.38 & 0.42 & NS & -0.29 & 0.39 & NS \\
\hline Android fat [kg] & -0.38 & 0.31 & -0.40 & NS & 0.30 & NS \\
\hline Gynoid fat [kg] & -0.31 & NS & NS & NS & NS & NS \\
\hline Android/gynoid fat & -0.40 & 0.35 & -0.42 & NS & NS & NS \\
\hline Trunk fat [kg] & -0.40 & 0.47 & -0.30 & -0.30 & 0.31 & -0.34 \\
\hline Trunk/total fat & -0.36 & 0.40 & NS & -0.29 & 0.33 & NS \\
\hline
\end{tabular}

${ }^{*}$ As measured by oral glucose tolerance test. ISI Matsuda and HOMA-IR indices in all groups. $M$ - metformin and diet group, $O$ - orlistat and diet group, BMI - body mass index, HOMA-IR - homeostatic model assessment-insulin resistance, ISI - insulin sensitivity index.

dominal to subcutaneous fat ratio [16, 17, 22]. To our knowledge, there are no studies that compare the effects of orlistat and metformin on obesity in terms of DXA measurements.

It should be noted that treatment with orlistat was associated with a reduction in body mass, $\mathrm{BMI}$ and body fat that was almost twice that seen in those treated with metformin. These results are in accordance with those obtained by Jayagopal et al. in their study of Caucasian women diagnosed with polycystic ovary syndrome (PCOS) [23]; however, they are contrary to Ghandi et al., who reported that women with PCOS treated with either drug showed a significant but comparable decline in body weight and waist circumference [24]. Metwally et al. observed similar effects on weight loss in obese anovulatory women after 3 months of orlistat or metformin treatment [25]. The weaker effect of metformin observed in our study may be attributable to a low daily dose of this medication, compared to previous studies. This hypothesis is supported by a meta-analysis of 14 studies of treatment with metformin, which showed that reduction in body mass depends on the daily dosage, and increases when it is higher than $1500 \mathrm{mg} /$ day [11]. Alternatively, this difference may be the result of the IR level in the study group.

On the basis of ISI Matsuda our study involved mainly insulin-sensitive women, whereas previous studies investigated women with PCOS integrally associated with IR, who benefited more from the insulin-sensitizing effect of metformin. Taking into consideration that Matsuda ISI is based both on fasting and post-load values of glucose and insu- 
lin, we may assess postprandial insulin resistance and predict cardiovascular risk more precisely [26].

Another possibility is that the greater effect of metformin observed in other studies may be related to the age of the study participants. In the study by Gokcel et al., women aged > 40 years, treated with metformin or orlistat, showed comparable reductions in BMI and waist circumference [27].

In our study, fasting insulin concentration did not change after 3 months of metformin or orlistat treatment. We observed a small, but not statistically significant, improvement in HOMA-IR indices. To date, knowledge of the efficacy of treatment with orlistat and metformin, and comparisons between the two, come mainly from studies of patients with PCOS. Our results align with those obtained by Jayagopal et al., who found no significant reduction in fasting insulin or HOMA-IR in women diagnosed with PCOS [22]. In contrast, Cho et al. found that, in women with PCOS, orlistat and metformin produced significant reductions in HOMA-IR [28]. Furthermore, in our study, comparable reductions in 120-min OGTT insulin levels and improvements in ISI Matsuda were observed in both groups. HOMA-IR is, to some extent, the equivalent of fasting serum insulin, as indicated by the strong correlation of these values [29]; therefore, it is important to test post-glucose-load insulin concentration using the OGTT. We used ISI Matsuda, which is highly correlated with the rate of whole-body glucose disposal during the euglycemic insulin clamp and corresponds better with metabolic disturbances [20]. Metformin and orlistat have different modes of action: the former acts through direct insulin sensitization, and the latter by decreasing fat absorption. In the metformin-treated group, insulin sensitivity, as measured by $120-\mathrm{min}$ insulin and ISI Matsuda, improved as a result of drug action. In the orlistat-treated group, the final effect was strengthened by a greater reduction in body fat with a further amelioration of insulin sensitivity.

Another important finding of this study was that high initial post-load insulin and low ISI Matsuda corresponded to reductions in waist, total and trunk fat (as measured by DXA) in both groups, as well as decreases in android fat and android/gynoid ratio in the metformin group. Stronger correlations between initial insulin resistance status and reduction in abdominal adiposity were found in the metformin group. Insulin-resistant individuals with greater visceral fat mass are typically regarded as being more prone to greater weight loss than other individuals having the same adiposity but without IR. This opinion is supported by the studies by Mediano et al., which demonstrated that IR facilitated weight loss in non-obese middle-aged women [30, 31]. Similar evidence comes from a 1-year lifestyle intervention, which augmented plasma glucose-insulin homeostasis in viscerally obese men, with corresponding improvements in SAT and VAT adiposity [32]. The results from other studies of the relationship between IR and weight change have been mostly observational and are still inconclusive. The study by Howard et al. demonstrated that IR was a predictor of weight increase [33]. In other studies the effectiveness of weight-loss therapy did not depend on differences in either initial IR or glycemic status $[34,35]$. However, these studies implemented only diet and exercise.

Finally, it is worth noting that both drugs were well tolerated and no serious adverse effects were observed in either group.

The main limitations of the study were the short duration of treatment and the small size of the study group. In addition, women with insulin resistance were not excluded. Another limitation is the open-label treatment; however, given that this was the first study of this kind, the data provided by the study are sufficient to justify the reassessment of longer treatment with higher doses, especially in the metformin group. Also a further double-blinded study should be designed.

In conclusion, treatment with orlistat resulted in a greater weight loss and improvement in body composition. Treatment with metformin caused a greater decrease of android fat. Orlistat and metformin produced similar improvement in insulin-glucose homeostasis. Insulin-resistant obese women demonstrated greater effects of treatment, irrespective of the drug used.

\section{Acknowledgments}

The research was supported in part by a grant from the Polish Ministry of Science and Higher Education (NN 404127438). English language assistance was provided by Proper Medical Writing Sp. z o.o.

\section{Conflict of interest}

The authors declare no conflict of interest.

\section{References}

1. Krzysztoszek J, Wierzejska E, Zielińska A. Obesity. An analysis of epidemiological and prognostic research. Arch Med Sci 2015; 11: 24-33.

2. Papaetis GS, Papakyriakou P, Panagiotou TN. Central obesity, type 2 diabetes and insulin: exploring a pathway full of thorns. Arch Med Sci 2015; 11: 463-82.

3. Lee CC, Glickman, SG, Dengel DR, Brown MD, Supiano MA. Abdominal adiposity assessed by dual energy X-ray absorptiometry provides a sex-independent predictor of insulin sensitivity in older adults. J Gerontol A Biol Sci Med Sci 2005; 60: 872-7. 
4. Petak S, Barbu CG, Yu EW, et al. The official positions of the International Society for Clinical Densitometry: body composition analysis reporting. J Clin Densitom 2013; 16: 508-19.

5. Hangartner TN, Warner S, Braillon P, Jankowski L, Shepherd J. The official positions of the International Society for Clinical Densitometry: acquisition of dual-energy X-ray absorptiometry body composition and considerations regarding analysis and repeatability of measures. J Clin Densitom 2013; 16: 520-36.

6. Josse AR, Atkinson SA, Tarnopolsky MA, Phillips SM. In creased consumption of dairy foods and protein during diet- and exercise-induced weight loss promotes fat mass loss and lean mass gain in overweight and obese premenopausal women. J Nutr 2011; 141: 1626-34.

7. Aucouturier J, Meyer M, Thivel D, Taillardat M, Duché P. Effect of android to gynoid fat ratio on insulin resistance in obese youth. Arch Pediatr Adolesc Med 2009; 163: 826-31.

8. Wiklund P, Toss F, Weinehall L, et al. Abdominal and gynoid fat mass are associated with cardiovascular risk factors in men and women. J Clin Endocrinol Metabol 2008; 93: 4360-6.

9. Toss F, Wiklund P, Nordström P, Nordström A. Body composition and mortality risk in later life. Age Ageing 2012; 41: 677-81.

10. Seifarth C, Schehler B, Schneider HJ. Effectiveness of metformin on weight loss in non-diabetic individuals with obesity. Exp Clin Endocrinol Diabetes 2013; 121: 227-31.

11. Nieuwenhuis-Ruifrok AE, Kuchenbecker WK, Hoek A, Middleton P, Norman RJ. Insulin sensitizing drugs for weight loss in women of reproductive age who are overweight or obese: systematic review and meta-analysis. Hum Reprod Update 2009; 15: 57-68.

12. Pasquali R, Gambineri A, Biscotti D, et al. Effect of longterm treatment with metformin added to hypocaloric diet on body composition, fat distribution, and androgen and insulin levels in abdominally obese women with and without the polycystic ovary syndrome. J Clin Endocrinol Metabol 2000; 85: 2767-74

13. Aghili R, Malek M, Valojerdi AE, Banazadeh Z, Najafi L, Khamseh ME. Body composition in adults with newly diagnosed type 2 diabetes: effects of metformin. J Diabetes Metab Disord 2014; 13: 88

14. Sjöström L, Rissanen A, Andersen T, et al. Randomised placebo-controlled trial of orlistat for weight loss and prevention of weight regain in obese patients. European Multicentre Orlistat Study Group. Lancet 1998; 352 167-72.

15. Moini A, Kanani M, Kashani L, Hosseini R, Hosseini L. Effect of orlistat on weight loss, hormonal and metabolic profiles in women with polycystic ovarian syndrome: a randomized double-blind placebo-controlled trial. Endocrine 2015; 49: 286-9.

16. Tiikkainen $M$, Bergholm R, Rissanen A, et al. Effects of equal weight loss with orlistat and placebo on body fat and serum fatty acid composition and insulin resistance in obese women. Am J Clin Nutr 2004; 79: 22-30.

17. Ozcelik O, Dogan H, Kelestimur H. Effects of a weight-reduction program with orlistat on serum leptin levels in obese women: a 12-week, randomized, placebo-controlled study. Curr Therap Res Clin Exp 2004; 65: 127-37.

18. Matthews DR, Hosker JP, Rudenski AS, Naylor BA, Treacher DF, Turner RC. Homeostasis model assessment: insulin resistance and beta-cell function from fasting plasma glucose and insulin concentrations in man. Diabetologia 1985; 28: 412-9.
19. deFronzo RA, Matsuda M. Reduced time points to calculate the composite index. Diabetes Care 2010; 33: e93.

20. Ganpule-Rao A, Joglekar C, Patkar D, et al. Associations of trunk fat depots with insulin resistance, beta-cell function and glycaemia - a multiple technique study. PLoS One 2013; 8: e75391.

21. Radikova Z, Koska J, Huckova M, et al. Insulin sensitivity indices: a proposal of cut-off points for simple identification of insulin-resistant subjects. Exp Clin Endocrinol Diabetes 2006; 114: 249-56.

22. Chanoine JP, Hampl S, Jensen C, Boldrin M, Hauptman J. Effect of orlistat on weight and body composition in obese adolescents: a randomized controlled trial. JAMA 2005; 293: 2873-83.

23. Jayagopal V, Kilpatrick ES, Holding S, Jennings PE, Atkin SL. Orlistat is as beneficial as metformin in the treatment of polycystic ovarian syndrome. J Clin Endocrinol Metabol 2005; 90: 729-33.

24. Ghandi S, Aflatoonian A, Tabibnejad N, Moghaddam $\mathrm{MH}$. The effects of metformin or orlistat on obese women with polycystic ovary syndrome: a prospective randomized open-label study. J Assist Reprod Genet 2011; 28: 591-6.

25. Metwally M, Amer S, Li TC, Ledger WL. An RCT of metformin versus orlistat for the management of obese anovulatory women. Hum Reprod 2009; 24: 966-75.

26. Lautt WW. Postprandial insulin resistance as an early predictor of cardiovascular risk. Ther Clin Risk Manag 2007; 5: 761-70.

27. Gokcel A, Gumurdulu Y, Karakose H, et al. Evaluation of the safety and efficacy of sibutramine, orlistat and metformin in the treatment of obesity. Diabetes Obes Metab 2002; 4: 49-55.

28. Cho LW, Kilpatrick ES, Keevil BG, Coady AM, Atkin SL. Effect of metformin, orlistat and pioglitazone treatment on mean insulin resistance and its biological variability in polycystic ovary syndrome. Clin Endocrinol 2009; 70: 233-7.

29. Kawada T. Preliminary report. Homeostasis model assessment of insulin resistance, an indicator of insulin resistance, is strongly related to serum insulin: practical data presentation and the mathematical basis. Metabolism 2010; 59: 1044-6.

30. Mediano MF, Sichieri R. Insulin resistance influences weight loss in non-obese women who followed a homebased exercise program and slight caloric restriction. Diabetes Res Clin Pract 2011; 92: 361-7.

31. Mediano MF, Sichieri R. Insulin resistance predicts the effectiveness of different glycemic index diets on weight loss in non-obese women. Obesity Facts 2012; 5: 641-7.

32. Borel AL, Nazare JA, Smith J, et al. Improvement in insulin sensitivity following a 1-year lifestyle intervention program in viscerally obese men: contribution of abdominal adiposity. Metabolism 2012; 61: 262-72.

33. Howard BV, Adams-Campbell L, Allen C, et al. Insulin resistance and weight gain in postmenopausal women of diverse ethnic groups. Int J Obes Relat Metab Disord 2004; 28: 1039-47.

34. McLaughlin T, Abbasi F, Carantoni M, Schaaf P, Reaven G. Differences in insulin resistance do not predict weight loss in response to hypocaloric diets in healthy obese women. J Clin Endocrinol Metabol 1999; 84: 578-81.

35. de Luis DA, Aller R, Izaola O, Gonzalez Sagrado M, Conde $R$. Differences in glycaemic status do not predict weight loss in response to hypocaloric diets in obese patients. Clin Nutr 2006; 25: 117-22. 